\title{
Improving the AODV Protocol to Satisfy the Required Level of Reliability for Home Area Networks
}

\author{
Hossein Jafari Pozveh \\ Department of Computer Engineering, Sheikhbahaee University, Baharestan, Isfahan, Iran \\ E-mail: hosein.jafari@gmail.com \\ Hossein Mohammadinejad and Mehdi Bateni \\ Department of Computer Engineering, Sheikhbahaee University, Baharestan, Isfahan, Iran \\ E-mail: \{mohammadi.n, bateni\}@ shbu.ac.ir
}

\begin{abstract}
For decades, the structure of existing power grids has not changed. It is an old structure that depends heavily on fossil fuel as an energy source, and in the future, this is likely to be critical in the field of energy. To solve these problems and to make optimal use of energy resources, a new concept is proposed, called Smart Grid. Smart Grid is an electric power distribution automation system, which can provide a two-way flow of electricity and information between power plants and consumers. The Smart Grid communications infrastructure consists of different network components, such as Home Area Network (HAN), Neighborhood Area Network (NAN) and Wide Area Network (WAN). Achieving the required level of reliability in the transmission of information to all sections, including the HAN, is one of the main objectives in the design and implementation of Smart Grid. This study offers a routing protocol by considering the parameters and constraints of HAN, which, by improving AODV routing protocol, achieves the level of required reliability for data transmission in this network. These improvements include: making table-driven AODV routing protocol, extending the routing protocol to compute multiple paths in a route discovery, simplification and providing the effect of HAN parameters. The results of the NS2 simulation indicate that applying this improved routing protocol in the HAN, satisfies the required level of reliability of the network, which is over $98 \%$.
\end{abstract}

Index Terms - Smart Grid, Home Area Network (HAN), Reliability, Routing, AODV Routing Protocol.

\section{INTRODUCTION}

A Smart Grid is an electricity supply network that controls all network activities and ensures the two-way flow of electricity and information among power plants and home appliances [1]. The two reasons for moving towards smart gird are: (1) the outdated current electric power system which needs to be improved to meet the future requirements and (2) the advantages of the Smart
Grid in the following five areas: environmental, economics, reliability, efficiency and security. According to the new studies conducted in converting the current electric power system into the Smart Grid, the design and implantation of an effective communications infrastructure for connection among components of the Smart Grid is of concern [2]. The Smart Grid components are: Home Area Network (HAN), Neighborhood Area Network (NAN) and Wide Area Network (WAN) which are named as the communication layers of Smart Grid. These layers and the correlation among them is illustrated in Fig. 1.

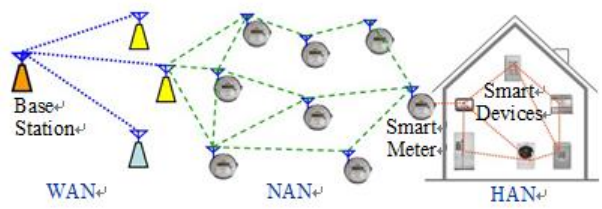

Fig.1. Communication layers of Smart Grid [3]

As observed in Fig. 1 HAN is the home area network that allows communication among electronics appliances and smart meters [4].

Quality of Service (QoS) is the capability of a network in providing certain services in terms of availability, bandwidth, reliability, delay, etc. QOS is very important in design and implantation of the Smart Grid. Smart Grid applications require high reliability in transmission of information which must be satisfied [2].

Packets in networks may be lost, altered or delayed during transmission, while the networks must be designed and implemented by considering these problems to ensure that each packet is sent to its destination in a timely and correct manner. The cause of many of these problems are: link disruptions, network congestions, and protocol errors [5].

Achieving the required level of reliability in transmission of information is one of the major challenges for HANs. With regard to one of the requirements of the HAN is providing reliability in data transmission, and given that an important factor in 
meeting these requirements is the use of routing protocol in HAN, the objective of this study is to improve ad hoc on-demand distance vector (AODV) routing protocol in order to satisfy the required level of reliability for the HAN part of the Smart Grid.

The rest of the paper is organized as follows: The related works are presented in section II; some basic definitions and concepts are presented in sections III and $\mathrm{IV}$; the proposed method for improving the AODV protocol to satisfy the required level of reliability for HANs is presented in section $\mathrm{V}$; the evaluation of the proposed routing algorithm is presented in section VI and the article is concluded in section VII.

\section{RELATED WORKS}

Since Smart Grid is a new technology, not enough studies are available regarding the subject of data transfer in this network. For this reason, this section surveys the works which related to increase reliability in wireless networks.

Mohapatra et al., [9] optimized the Reverse Ad hoc On-demand Distance Vector (R-AODV) based on computing the Reliability Factor (RF) of nodes. This factor includes route stability and energy aware metric. The proposed protocol uses two paths to send packets, primary path with high RF and secondary path with descending order of RF. Chughtai et al., [10] proposed a technique in routing, which selects a path from source node to destination node with the least congestion. In this technique each node computes the traffic load based on the Packet Delivery Ratio and hop count metrics, then the path with the minimum traffic load is selected. Zhu et al., [11] improved the AODV routing protocol, which uses a secondary path when data packets cannot be delivered through the main path. Zhang et al., [12] proposed a multi-path routing protocol, which finds the first shortest and the second shortest paths. These two paths are independent of each other. Ren and $\mathrm{Wu}$ [13] proposed a method, where every node in network must be covered by more than one coordinator node. Although this method provides more reliable network, it is costly. Li et al., [14] proposed a multi-path routing protocol, where two paths are used at the same time. When a failure occurs on the primary path, this protocol uses a bridge to transfer the data to the other path, and improves the reliability of transmission in a significant manner. Valarmathi and Malmurugan [15] proposed a multipath routing protocol, which selects a path from source node to destination node with the least interference. The combined metric is computed based on the interference and load of each link. The source node finds the path with the minimum value of the combined metric. When the failures occur in the primary route, the alternate route is chosen with descending order of combined metric value. Kim and Ngo [16] proposed a routing protocol, which finds the route with the highest packet delivery ratio to transmit data packets. Zhong et al., [17] proposed a routing protocol for Zigbee wireless network, which combines Cluster-Tree and AODVjr routing protocols. The
AODVjr routing protocol is applied for routing discovery and Cluster-Tree protocol is applied for fast routing. Aron et al., [18] improved the AODV routing protocol, which applies the backup route technique. If there is a failure in primary path, the backup routes are applied. Jawhar et al., [19] proposed a routing protocol, which is based on the DSR protocol. In this protocol each node maintains a reliability factor. During route discovery process, an intermediate node only propagates the request messages to other nodes with a reliability factor above the needed threshold. Marina and Das [20] extended the AODV routing protocol, which finds multiple link-disjoint and loop-free routes.

\section{Classification Of Ad Hoc Routing Protocols}

Whenever a packet is to be sent from a source node to a destination node by intermediate nodes, routing protocols are responsible for finding paths from source node to destination node. These routing protocols are categorized as follows:

\section{A. On-demand Routing Protocols}

In this category the routes are established upon necessity. The source node begins a route discovery process whenever it needs to send data towards destination node. When a route or routes are found, the discovery process is considered as complete. When routes are created, the procedure of route maintenance is followed, which maintains valid routes and eliminates invalid routes. Different on-demand routing protocols consist of AODV, Dynamic Source Routing (DSR), etc. [6].

AODV is an on-demand routing protocol. The discovery process is OFF until a route is required. Upon demand, the source node which needs the route, broadcasts a request packet to its neighbors. When an intermediate node receives a request packet and it has an entry corresponding to the destination node address in its routing table, it returns a reply packet through the reverse path to the source node; otherwise, rebroadcasts the request packet to its neighbors. This procedure will continue until an intermediate node can return a reply packet through the reverse path to the source node or the request packet reaches the destination node and destination node returns a reply packet through the reverse path. Next, the source node recognizes a new path and transmits its data through this path to the destination node [7].

\section{B. Table-Driven Routing Protocols}

This category of protocols holds the routing information from each node to every other nodes in the network, regardless of whether these routes are needed or not. Every node in the network holds a table, known as routing table, where routing information is maintained and assists nodes to get the route information and establish a path. Different table-driven routing protocols consist of Destination Sequenced Distance Vector (DSDV), Optimized Link State Routing (OLSR), etc. [6]. 


\section{WIRELESS TECHNOLOGIES FOR USE IN HANS}

This section surveys two different wireless technologies for implementation of HAN. ZigBee and $\mathrm{Wi}-\mathrm{Fi}$ are the technologies of concern in this article.

\section{A. ZigBee}

ZigBee is a wireless technology built on the IEEE 802.15.4 standard. It supports low data rate, which vary from $40 \mathrm{kbps}$ in the $915 \mathrm{MHz}$ band up to $250 \mathrm{kbps}$ in the $2.4 \mathrm{GHz}$ band. ZigBee can be applied in Personal Area Networks (PANs), home and building automation, etc. This wireless technology supports 10-75 meters line-of-sight and about 30 meters in indoor environment. In a mesh network each node can self-route and connect with other nodes. For this reason, devices which use ZigBee technology can transmit data over unlimited distances with mesh networking. These characteristics make ZigBee technology appropriate for the HAN applications [8].

\section{B. Wi-Fi}

Wi-Fi is a wireless technology built on the IEEE 802.11 standard. It supports maximum coverage distances of 250 meters and maximum data rates of $150 \mathrm{Mbps}$. Wi-Fi can be applied in home automation, laptops, smart phones and other electronic devices. Wi-Fi technology is an appropriate choice in networks in low interference environments and small data rate requirements. These characteristics make Wi-Fi technology appropriate for the HAN applications [8].

\section{PROPOSED METHOD}

The proposed method in this article is to improve the AODV routing protocol in order to be applied in the HAN and to reach the required level of reliability. This method is outlined in Fig. 2. The obtained routing protocol is named Ad hoc Table-driven Distance Vector Multipath (ATDVM). The procedure in this method consists of three main steps:

\section{A. Making a table-driven AODV routing protocol}

As mentioned in section III, the AODV routing protocol is an on-demand routing protocol, where the routes are created upon necessity. On the contrary, table-driven routing protocols perform better in small networks with low mobility, such as HANs; therefore, the first step of this proposed method is to make a change in AODV routing protocol from on-demand mode to table-driven mode. This change requires the network to be configured prior to the information transfer. Before the nodes make a request to send information, each node must create a path to the smart meter and save it in its routing table. Since the paths in HANs are bidirectional, the smart meter must create paths to all nodes in the network and store them in its routing table. The point here is, whether paths are needed or not, they must be created and stored in the routing tables. After the configuration is completed and the paths are saved in the routing tables, the nodes can send data to the destination node. The pseudo-code for making the table-driven AODV routing protocol is presented as follows, the Algorithm 1 shows the pseudo-code for discovering the paths from each node to the smart meter and storing them in routing tables, and the Algorithm 2 shows the pseudo-code for discovering paths from the smart meter to each node in the network, then storing them in its routing table.

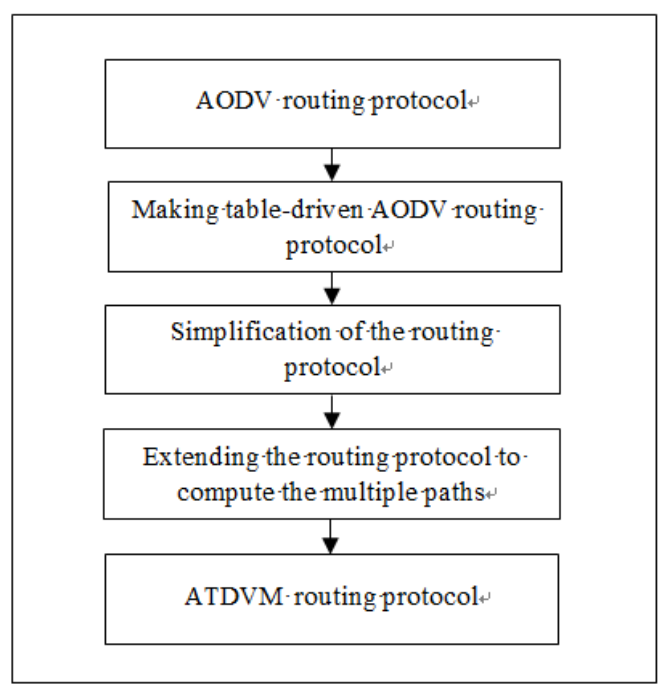

Fig.2. Proposed Method for Improving AODV Routing Protocol

\section{B. Simplification}

After designing the table-driven AODV routing protocol, its simplification is an essential. In AODV routing protocol, hello messages are applied to detect link failures. These failures can be detected by applying Link Layer ACKnowledgments (LLACKs) as well [18]. Periodic hello messages at specified intervals, are applied more commonly for networks with high node mobility. In such cases, the hello messages are applied to identify link failures rapidly, execute route discovery procedures and update the routing tables. Due to the low mobility of nodes in HANs, link failures occur less often; therefore, in this network, hello messages are not required for updating paths and LLACKs are applied instead.

\section{Extending the routing protocol to compute multiple paths in a route discovery}

Using multiple alternative paths in a network can yield a variety of advantages, overcome the drawbacks of unipath routing protocols and enhance network utility in terms of routing efficiency, packet delivery ratios, fault tolerance, etc. Since applying multiple paths will enhance reliability, the routing protocol after being adapted to be table-driven and simplification, is extended to compute multiple paths in a route discovery. The computed multiple routes can be overlapped, link-disjointed or node-disjointed. In this proposed method, the obtained routing protocol is extended to multipath link-disjoint form. The discovery of more paths in link-disjoint form compared to that of the node-disjoint, makes multipath link-disjoint preferable. 
Algorithm 1. Pseudo-code for discovering the paths from each node to the smart meter

Find_Route from Source to target and update Route Table Input:
$S$, Source ID
$D$, Destination ID
$R$, Route Table

Output: Updated Route Table

\section{Method:}

(1) Create Pointer To $R$

(2) For each $S$ in network do

(3) \{

(4) Generate Request Packet

(5) Set $r q_{-}$des to $D$

(6) Set $r q_{-} s r c$ to $S$

(7) Set $r q \_$type to RREQ

(8) Increase BROADCAST ID

(9) Broadcast Request Packet

(10) \}

(11) Update $R$ with new Path(s) detected

(12) Return:

Algorithm 2. Pseudo-code for discovering paths from the smart meter to each node in the network

Reverse_Route, Route from Destination to any Source and update Route Table

Input:
$D$, Destination ID
$S$, Source ID
$R$, Route Table

Output: Updated Route Table

Method:

(1) Create Pointer To $R$

(2) For each $S$ in network do

(3) \{

(4) Generate Request Packet

(5) Set $r q \_d e s$ to $S$

(6) Set $r q_{-} s r c$ to $D$

(7) Set $r q \_$type to RREQ

(8) Increase BROADCAST ID

(9) Broadcast Request Packet

(10) \}

(11) Update $R$ with new Path(s) detected

(12) Return;

\section{RELIABILITY ANALYSIS OF ATDVM ROUTING PROTOCOL}

This section, first, describes the simulation parameters applied in this study and then evaluates the reliability of ATDVM protocol on the NS2 platform and compares it with the Ad hoc On-demand Distance Vector Multipath (AODVM) and the AODV protocols.

\section{A. Simulation Settings}

Network simulation parameters are presented in Table1. The shadowing propagation model is adopted with soft partition and hard partition environment characteristics for simulating packet loss and obtaining realistic results. The ATDVM, AODVM and AODV routing protocols are applied as the routing layer protocols, with each node sending Constant Bit Rate (CBR) traffic of 40 bytes to sink node.

Table 1. Network Simulation Parameters

\begin{tabular}{|c|c|}
\hline Parameter & Value \\
\hline Simulator & NS-2.35 \\
\hline Environment Size & $40 \times 40 \mathrm{~m}^{2}$ \\
\hline Number of Nodes & 20 \\
\hline Simulation Time & CBR seconds \\
\hline Traffic Type & TCP \\
\hline Transport Layer & 40 bytes \\
\hline CBR Packet Size & 1 second \\
\hline CBR Packet Interval & 100 Kbps \\
\hline Bandwidth & $802.11,802.15 .4$ \\
\hline MAC Layer & Shadowing \\
\hline PHY Layer & Path Loss Exponent $=1.6$ \\
\hline Propagation Model & Shadowing deviation $=9.6$ \\
\hline Soft Partition Parameters & Path Loss Exponent $=4$ \\
\hline Hard Partition Parameters & Shadowing deviation $=7$ \\
\hline Interface Queue Size & 50 Packets \\
\hline Routing Protocols & ATDVM, AODVM, AODV \\
\hline
\end{tabular}

\section{B. Simulation Results and Discussion}

The reliability of information transmission is evaluated through Packet Delivery Ratio (PDR) metric. PDR is the ratio of number of received data packets to number of sent data packets. This ratio represents the reliability of the network. For the HAN, this is the most important metric in measuring electricity consumption. The obtained results of this study consist of the following 5 assessments with different parameters:

1) PDR versus number of source nodes with ZigBee in soft partition environment

As observed in Fig. 3, when multiple nodes send packets to the destination node in a simultaneous manner, the ATDVM routing protocol outperforms AODVM and AODV routing protocols. The PDR of AODV protocol is severely degraded with an increase in the number of source nodes.

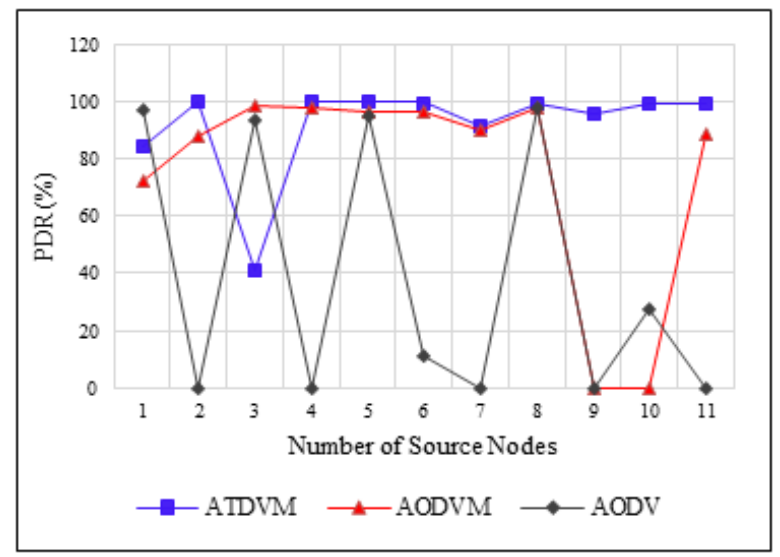

Fig.3. PDR Versus Number of Source Nodes with ZigBee in Soft Partition

2) PDR versus number of source nodes with Wi-Fi in soft partition environment 
As observed in Fig. 4, when multiple nodes simultaneously send packets to the destination node in a simultaneous manner, the ATDVM routing protocol usually performs better than AODVM and AODV routing protocols. The PDR of ATDVM protocol remains close to $100 \%$, even when the number of source nodes is increased. On the other hand, the PDR of AODV protocol is degraded with an increase in the number of source nodes.

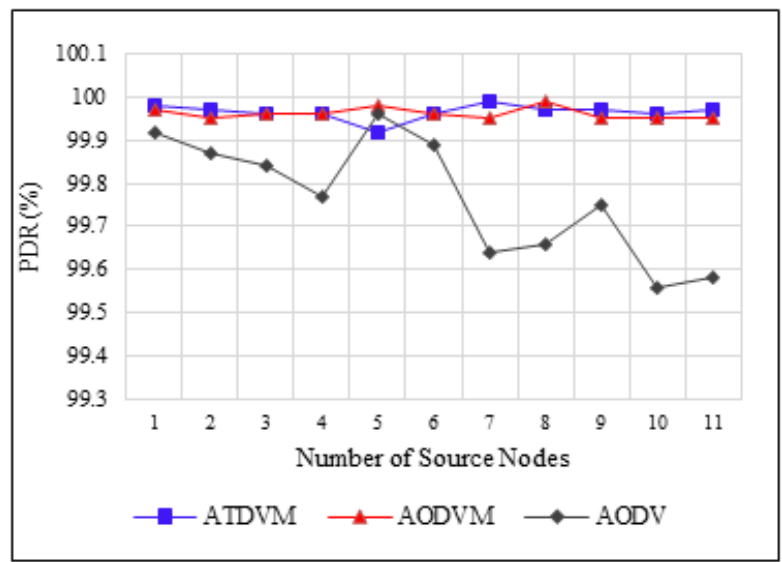

Fig.4. PDR Versus Number of Source Nodes with Wi-Fi in Soft Partition

3) PDR versus number of source nodes with Wi-Fi in hard partition environment

As observed in Fig. 5, when the shadowing propagation model is adopted with hard partition characteristics, the ATDVM protocol always outperforms the AODVM protocol and most of the time it outperforms the AODV protocol.

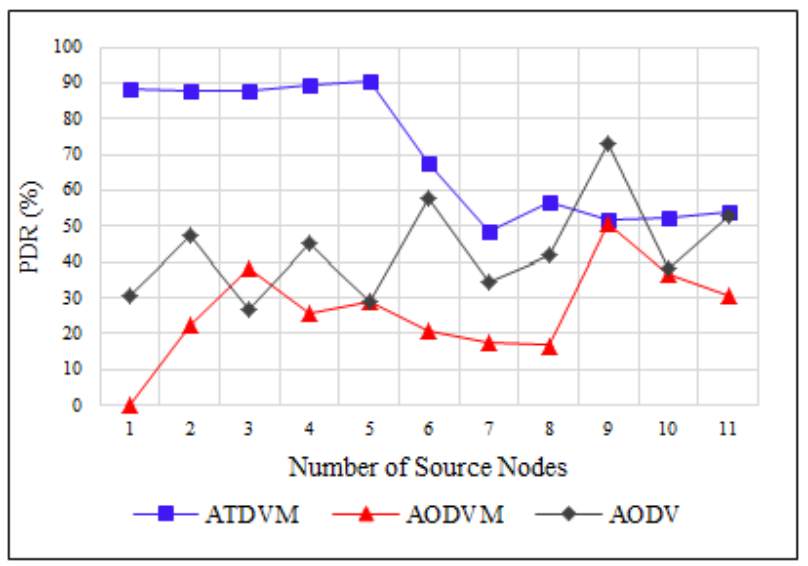

Fig.5. PDR Versus Number of Source Nodes with Wi-Fi in hard Partition

4) PDR versus number of mobile nodes with ZigBee in soft partition environment

The simulation of this section has been done with one source node. As observed in Fig. 6, when multiple nodes move in a simultaneous manner in the HAN, the ATDVM protocol outperforms the AODVM and the AODV protocols.

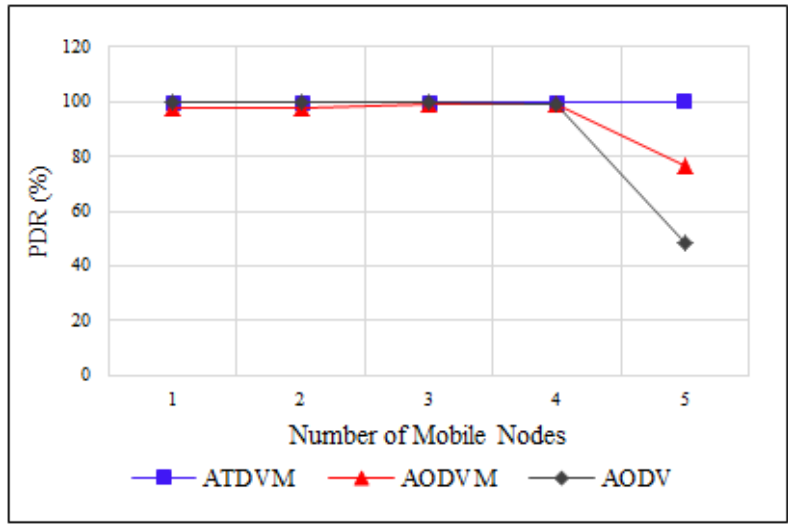

Fig.6. PDR Versus Number of Mobile Nodes with ZigBee in Soft Partition

5) PDR versus number of offline nodes with ZigBee in soft partition environment

The simulation of this section has been done with one source node. As observed in Fig. 7, when multiple nodes become offline in the HAN in a simultaneous manner, the ATDVM routing protocol outperforms the AODVM and AODV protocols. The PDR in ATDVM and AODVM protocols is degraded with an increase in the number of offline nodes, however, the PDR of ATDVM protocol is still higher than that of AODVM and AODV protocols.

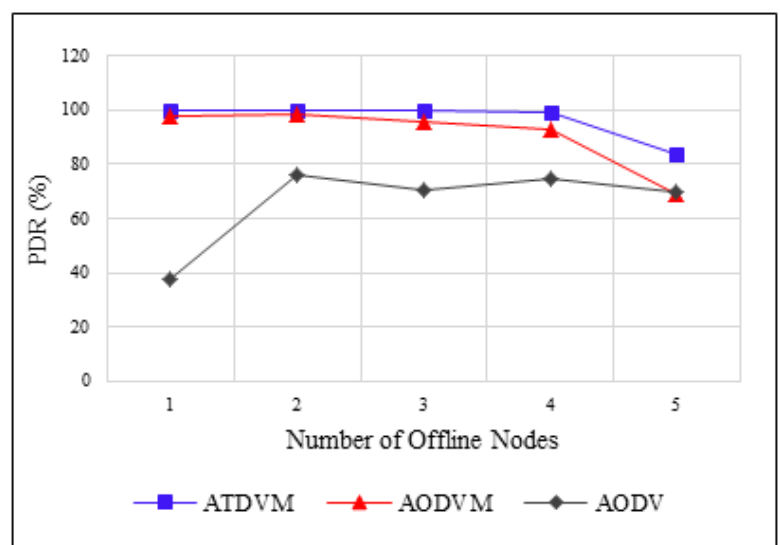

Fig.7. PDR Versus Number of Offline Nodes with ZigBee in Soft Partition

\section{CONCLUSION AND FUTURE SCOPE}

Satisfying the required level of reliability in information transmission in HAN is one of the important issues involved in implementing Smart Grid communications. The required level of reliability in the HAN is satisfied with respect to the specifications of HAN, improving and adapting AODV routing protocol to be applied in HAN. This newly improved routing protocol is named ATDVM. The simulation results indicate that the ATDVM protocol outperforms the AODV and AODVM protocols in terms of PDR and reliability. Moreover, in order to yield the required level of reliability of HAN, which is over $98 \%$, it is possible to 
apply ATDVM as the routing layer protocol and the Wi-Fi (IEEE 802.11) as the physical and MAC layers.

The future work will focus on achieving the required level of reliability in the information transmission to NAN, where by considering the parameters and constraints of NAN, AODV protocol can be improved to satisfy the required level of reliability for NANs.

\section{ACKNOWLEDGMENT}

Our special thanks is extended to Sheikhbahaee University faculty for contributing to the development of this research.

\section{REFERENCES}

[1] J. Tripathi, J. C. de Oliveira, and J. P. Vasseur, "Applicability Study of RPL with Local Repair in smart grid Substation Networks," 2010 First IEEE International Conference on smart grid Communications, Oct. 2010.

[2] N. Saputro, K. Akkaya, and S. Uludag, "A survey of routing protocols for smart grid communications," Computer Networks, vol. 56, no. 11, pp. 2742-2771, Jul. 2012.

[3] Q.-D. Ho and T. Le-Ngoc, "smart grid Communications Networks: Wireless Technologies, Protocols, Issues, and Standards," Handbook of Green Information and Communication Systems, pp. 115-146, 2013.

[4] V. Namboodiri, V. Aravinthan, S. N. Mohapatra, B. Karimi, and W. Jewell, "Toward a Secure Wireless-Based Home Area Network for Metering in smart grids," IEEE Systems Journal, vol. 8, no. 2, pp. 509-520, Jun. 2014.

[5] W. Wang, Y. Xu, and M. Khanna, "A survey on the communication architectures in smart grid," Computer Networks, vol. 55, no. 15, pp. 3604-3629, Oct. 2011.

[6] B. Bhatia, N. Sood, "AODV based Congestion Control Protocols: Review," International Journal of Computer Science and Information Technologies, Vol. 5 (3), 2014.

[7] M. Kasraoui, A. Cabani, and J. Mouzna, "Improvement of Zigbee Routing Protocol," 2012 IEEE International Conference on Green Computing and Communications, Nov. 2012.

[8] A.Mahmood, N. Javaid, and S. Razzaq, "A review of wireless communications for smart grid," Renewable and Sustainable Energy Reviews, vol. 41, pp. 248-260, Jan. 2015.

[9] S. K. Mohapatra, B. R. Swain, S. K. Mahapatra, and S. K. Behera, "Stability and energy aware reverse AODV routing protocol in MANETS," 2015 IEEE 2nd International Conference on Recent Trends in Information Systems (ReTIS), Jul. 2015.

[10] O. Chughtai, N. Badruddin, A. Awang, and M. Rehan, "Reliability-constrained routing for traffic load balancing in Wireless Sensor Networks," 2014 5th International Conference on Intelligent and Advanced Systems (ICIAS), Jun. 2014.

[11] Lin Zhu, Chuang Lin, Kun Meng, and Yangwei Dong, "P-AODV: A protection routing mechanism in wireless mesh networks," 2013 15th IEEE International Conference on Communication Technology, Nov. 2013.

[12] Changzhong Zhang, Siyuan Liu, Zhuo Sun, and Shaofan Sun, "A breadth-first and disjoint multi-path routing algorithm in wireless mesh networks," 2013 15th IEEE International Conference on Communication Technology, Nov. 2013.

[13] Ren, Yu, and Kelong Wu, "The Zigbee Network Model with Reliable Communication," Proceedings of International Conferences NGCIT, 2013.

[14] J. Li, L. Zhang, L. Guo, and L. Yao, "A path-transfer based multi-path reliable routing in wireless sensor networks," 2012 IEEE 31st International Performance Computing and Communications Conference (IPCCC), Dec. 2012.

[15] K. Valarmathi and N. Malmurugan, "Multi path routing protocol for Improving Reliability in IEEE 802.16 Wireless Mesh networks," 3rd International Conference on Trendz in Information Sciences \& Computing (TISC2011), Dec. 2011.

[16] Myung Kyun Kim and H. P. Ngo, "A reliable and energy efficient routing protocol in industrial wireless sensor networks," The 2011 International Conference on Advanced Technologies for Communications (ATC 2011), Aug. 2011.

[17] D. Zhong, W. Ji, Y. Liu, J. Han, and S. Li, “An improved routing algorithm of Zigbee wireless sensor network for smart home system," The 5th International Conference on Automation, Robotics and Applications, Dec. 2011.

[18] Z. Che-Aron, W. F. M. Al-Khateeb, and F. Anwar, "ENFAT-AODV: The fault-tolerant routing protocol for high failure rate Wireless Sensor Networks," 2010 2nd International Conference on Future Computer and Communication, 2010.

[19] I. Jawhar, Z. Trabelsi, and J. Al-Jaroodi, "Towards More Reliable Source Routing in Wireless Networks," 2008 International Conference on Networking, Architecture, and Storage, Jun. 2008.

[20] M. K. Marina and S. R. Das, "On-demand multipath distance vector routing in ad hoc networks," Proceedings Ninth International Conference on Network Protocols. ICNP 2001, 2001.

[21] C. Perkins, E. Belding-Royer, and S. Das, " Ad hoc On-Demand Distance Vector (AODV) Routing," Jul. 2003.

\section{Authors' Profiles}

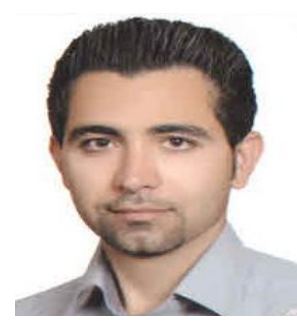

Hossein Jafari Pozveh received his B.Sc. degree in Software Engineering from Faculty of Engineering, Islamic Azad University of Isfahan (Khorasgan), Isfahan, Iran in 2013. He is now an M.Sc. Student of Department of Computer Engineering at Sheikhbahaee University, Isfahan, Iran. His research interests include reliability in Wireless Network and Smart Grid.

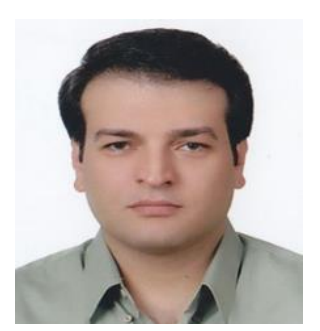

Hossein Mohammadinejad received the B.Sc. degree in computer engineering in 2000 from University of Tehran, Tehran, Iran, and the M.Sc. degree in computer engineering from University of Isfahan, Isfahan, Iran, in 2003. He is currently working toward a Ph.D. degree with the Department of Computer Engineering, University of Isfahan, Isfahan, Iran. He has worked as a Lecturer at Sheikhbahaee University, Isfahan, Iran, since 2003. 


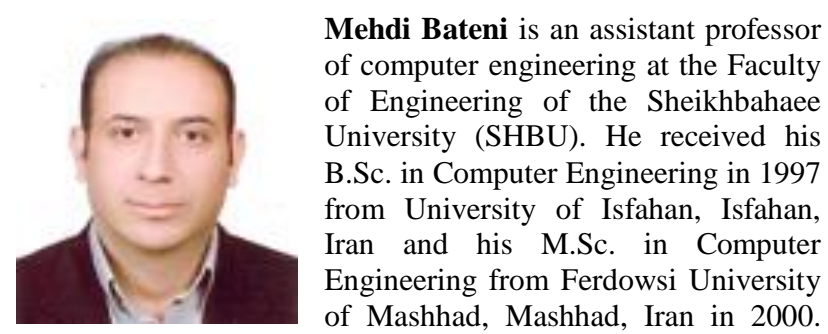

He received his Ph.D. in Computer Engineering in 2012 from University of Isfahan, Isfahan, Iran.

How to cite this paper: Hossein Jafari Pozveh, Hossein Mohammadinejad, Mehdi Bateni,"Improving the AODV Protocol to Satisfy the Required Level of Reliability for Home Area Networks", International Journal of Computer Network and Information Security(IJCNIS), Vol.8, No.6, pp.22-28, 2016.DOI: 10.5815/ijcnis.2016.06.03 